\title{
Kindergartens Reopening in the Period of Regular Epidemic Prevention and Control, Benefitial or Harmful?*
}

\author{
Qiao-qiao DONG ${ }^{1 \dagger}$, Li-ru QIU ${ }^{1 \dagger}$, Li-ming $\mathrm{CHENG}^{2}$, Sai-nan $\mathrm{SHU}^{1}$, Yu CHEN ${ }^{1}$, Yue ZHAO ${ }^{1}$, Yan $\mathrm{HAO}^{1}$, $\mathrm{Huan}_{\mathrm{SHI}}{ }^{1}$, \\ Xiao-ping $\mathrm{LUO}^{1 \#}$ \\ ${ }^{1}$ Department of Pediatrics, Tongji Hospital, Tongji Medical College, Huazhong University of Science and Technology, Wuhan \\ 430030, China \\ ${ }^{2}$ Department of Laboratory, Tongji Hospital, Tongji Medical College, Huazhong University of Science and Technology, Wuhan \\ 430030, China
}

(C) Huazhong University of Science and Technology 2020

\begin{abstract}
Summary: In the period of regular epidemic prevention and control of coronavirus disease 2019 (COVID-19) in our country, work resumption has been fully advanced. But there are still new sporadic local cases and imported cases across the country. In this situation, whether kindergartens reopening will increase the risk of severe acute respiratory syndrome coronavirus 2 (SARS-CoV-2) spread still remains uncertain. We reviewed two pediatric patients with moderate COVID-19, collected the epidemiologic information and monitored the cycle threshold value of rectal specimen and the viral loads, and discussed the transmission of SARS-CoV-2 in pediatric patients and the virulence of feces in children with moderate COVID-19, in order to analyze the risk of kindergartens reopening.
\end{abstract}

Key words: COVID-19; SARS-CoV-2 transmission; children; viral load

The outbreak of coronavirus disease 2019 (COVID-19) is a global pandemic ${ }^{[1,2]}$. Adult-to-adult and adult-to-child transmission of the severe acute respiratory syndrome coronavirus 2 (SARS-CoV-2) from patients or asymptomatic carriers has been confirmed $^{[3-5]}$. As of September 11, 2020, more than 7 million people had been reported to be infected worldwide, and the total number of confirmed infected people is up to more than 28 million. In our country, after the 76-day lockdown of Wuhan and several months of regular epidemic prevention and control, the epidemic has basically been brought under control. Even so, there are still new sporadic cases, imported cases and asymptomatic carriers that have been found every day.

At present, the staged results of epidemic prevention and control in our country have been further consolidated, important progress has been made in work resumption, and the restoration of economic and social order has accelerated. In these circumstances,

Qiao-qiao DONG, E-mail: doctordong2016@163.com; Liru QIU, E-mail: liruqiutj@163.com

${ }^{\dagger}$ The authors contributed equally to this study.

\#Corresponding author, E-mail: xpluo@tjh.tjmu.edu.cn

*This study was supported by funds from Tongji Hospital of Tongji Medical College, Huazhong University of Science and Technology (No. XXGZBDYJ005) and Wuhan Science and Technology Bureau (No. 2020020601012228). primary and secondary schools and kindergartens across the country have started one after another.

It has been proved that children are also susceptible to SARS-CoV-2 and most pediatric patients are diagnosed with asymptomatic, mild or moderate disease ${ }^{[6-10]}$, and therefore much easier to be missed $^{[11]}$. Moreover, it's more difficult for toddlers to wear masks, especially without the close supervision of parents. In this situation, whether schools reopening especially kindergartens reopening will increase the risk of epidemic spread needs to be discussed.

In our study, we reviewed the epidemiologic information of two pediatric patients with moderate COVID-19 and monitored the cycle threshold $(\mathrm{Ct})$ value of rectal specimen and the viral loads, analyzing the transmission of SARS-CoV-2 in pediatric patients, in order to discuss the risk of kindergartens reopening.

\section{MATERIALS AND METHODS}

Two pediatric patients were enrolled and their detailed epidemiological and clinical data were collected. All examinations and investigations in the two cases were approved by the Ethical Committee of Tongji Hospital of Huazhong University of Science and Technology (China) (TJ-C20200359) and were conducted in accordance with the Declaration of Helsinki. Written informed consent was obtained from 
the parents or legal guardians of the recruited patients.

Nasopharyngeal and rectal swabs of the two patients were collected daily during the hospitalization. Blood samples of the patients were taken at admission, during hospitalization and on discharged day. Chest computed tomographic (CT) images were collected when the patients were diagnosed.

The reverse transcriptase polymerase chain reaction (RT-PCR) tests of SARS-CoV-2 nucleic acid of nasopharyngeal and rectal specimen were performed, using fully automated Nucleic Acid Extraction System 9600E (Xi' an TianLong Science and Technology Co., Ltd., China). Swabs were collected from the patients and placed immediately in $3 \mathrm{~mL}$ viral transport media (VTM) and transported to the laboratory. Total nucleic acid was extracted from $200 \mu \mathrm{L}$ of specimen. Diagnosis of SARS-CoV-2 infection was made using real-time RT-PCR in the laboratory. Two target genes were simultaneously amplified and tested, including open reading frame $1 \mathrm{ab}(\mathrm{ORF} 1 \mathrm{ab})$ and nucleoprotein (N). The following primers were used for the detection of ORF1ab gene and N gene of SARS-CoV-2 (http:// ivdc.chinacdc.cn/kyjz/202001/t20200121_211337. $\mathrm{html}$ ): for ORF1ab gene, forward primer, CCCTGTGGGTTTTACACTTAA, reverse primer, ACGATTGTGCATCAGCTGA, the probe, 5'-FAM-CCGTCTGCGGTATGTGGAAAGGTTATGG-BHQ1-3'; for N gene, forward primer, GGGGAACTTCTCCTGCTAGAAT, reverse primer, CAGACATTTTGCTCTCAAGCTG, the probe, 5'-FAM-TTGCTGCTGCTTGACAGATTTAMRA-3'.

Clinical diagnostic kit (DAAN Gene Co., Ltd, China) approved by China Food and Drug Administration (CFDA) was applied. A Ct value $\leq 40$ was defined as positive and the cases were confirmed as COVID-19. A Ct value $>40$ was defined as negative.

The SARS-CoV-2 viral load of nasopharyngeal and rectal specimen was detected using a novel coronavirus nucleic acid detection kit (Applied Biological Technologies Co., Ltd., China). The standard material was low-concentration novel coronavirus nucleic acid standard material from National Institute of Metrology (China). The $\mathrm{N}$ gene concentration of the standard solution was 9800 copies $/ \mu \mathrm{L}$ with a total of 6 concentration gradients. The reaction solution was prepared according to the instructions of the test kit. The qPCR reaction was performed on Thermo Fish's ABI 7500 quantitative PCR instrument. The viral load was calculated using the $\mathrm{Ct}$ value of $\mathrm{N}$ gene.

CFDA approved clinical diagnostic kits of 2019$\mathrm{CoV}$ antibody IgM\&IgG (YHLO Biotech Co., Ltd., China) were used in blood SARS-CoV-2 antibody tests. The iFlash-SARS-CoV-2 IgG assay was a paramagnetic particle chemiluminescent immunoassay (CLIA) for qualitative determination of IgG and IgM antibody to SARS-CoV-2 in human serum and plasma using the iFlash Immunoassay Analyzer.

\section{RESULTS}

Patient 1 , female, aged 2 years and 5 months old, had dinner with her family at a mall restaurant in Wuhan on January 18 th, 2020. She initially developed fever and dry cough on January 31 st. She was diagnosed as moderate COVID-19 on February 5th in Tongji Hospital of Wuhan according to the chest CT images and positive rectal nucleic acid swab test. She was asked to be quarantined at home and alleviated on February 8th. Her nucleic acid test of rectal swab on March 5th was still positive and she was subsequently hospitalized. On March 21st her nucleic acid test of rectal swab turned negative and she was finally discharged on April 4th (fig. 1).

Patient 2, female, aged two years and four months. Her mother had fever on January 18th, 2020 and was diagnosed as COVID-19 on January 25th. Subsequently, patient 2 ran a fever on February 5 th and diagnosed as moderate COVID-19 in Tongji Hospital of Wuhan based on the chest CT images and positive nasopharyngeal nucleic acid test on February 9th. She was hospitalized on February 15th. Her nucleic acid test of rectal swab turned negative on March 26th, and she was discharged on April 1st (fig. 1).

\subsection{Epidemiological Investigation}

Patient 1 had been living at home with her family and was in close contact with her 7-year-old sister, parents and grandparents before onset and after being diagnosed. Since January 31 st, the day that patient 1 had fever, she had been quarantined at home and her family had started to wear masks. Parents slept with her at night, without any protection. Her sister occasionally played toys with her. During hospitalization, her mother took care of her and also slept without masks. All these close contacts of patient 1 had no symptoms and their chest CT images, nucleic acid tests of nasopharyngeal and rectal swabs and serum IgM and IgG antibody of SARS-CoV-2 were all negative.

Patient 2's mother was diagnosed with COVID-19 and her father was an asymptomatic carrier. They were both quarantined in hospital. Patient 2 had been living and sleeping with her grandmother since January 18th. Neither of them wore a mask throughout the onset and hospitalization of patient 2. The nucleic acid tests, IgM and IgG antibody and chest CT images of her grandmother were proved to be all negative (fig. 1).

\subsection{Symptoms}

Patient 1 presented with fever and dry cough on January 31 st and had a maximum temperature of $39.7^{\circ} \mathrm{C}$. Vomiting was occasionally observed after drinking milk. She had no other respiratory symptoms or gastrointestinal symptoms, including shortness of breath, dyspnea and diarrhea, etc. On February 6th 


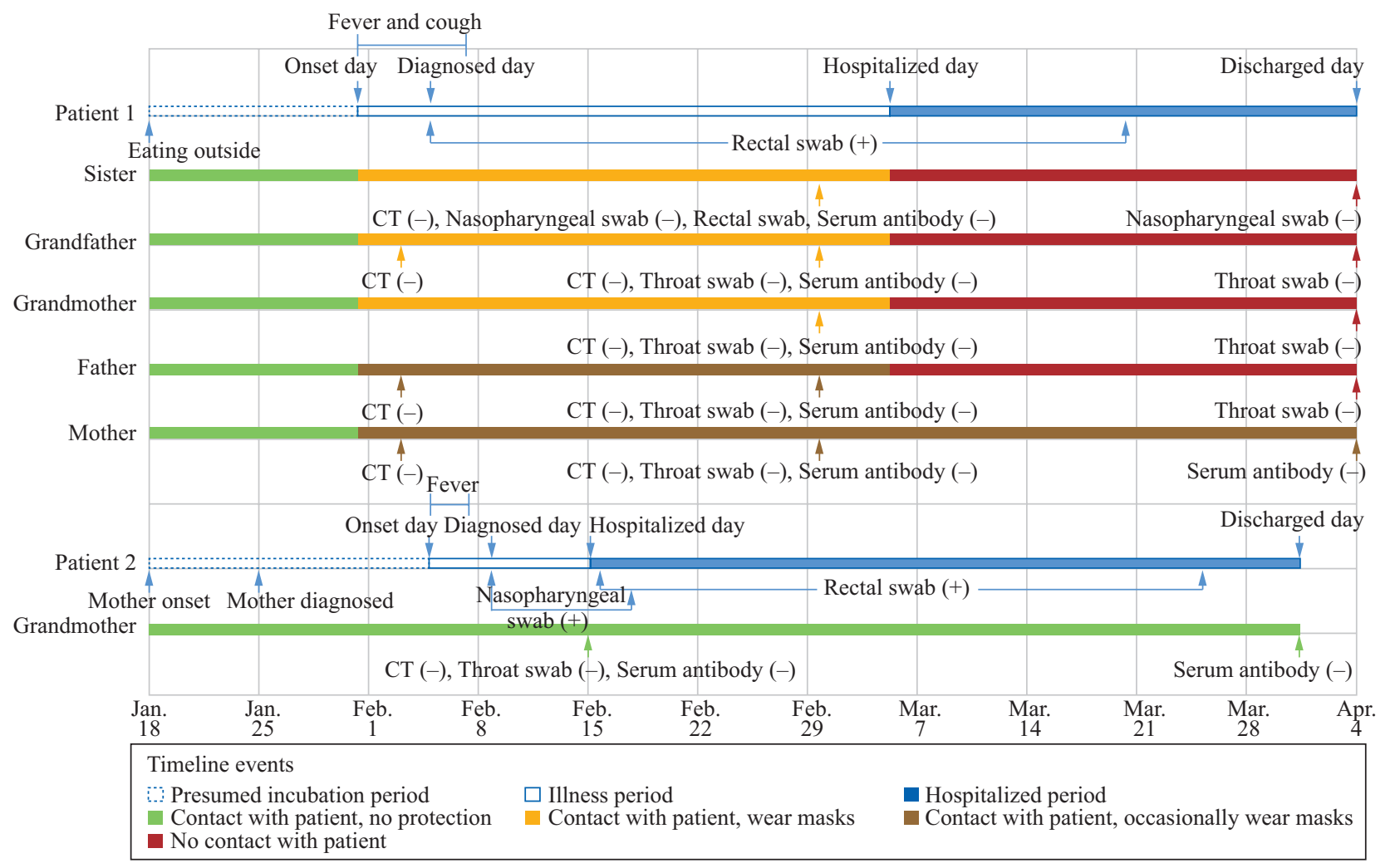

Fig. 1 The clinical courses of patient 1 and patient 2

the peak temperature decreased to $38.7^{\circ} \mathrm{C}$. Fever and cough lasted for 8 days. Since February 8th, she has been remained asymptomatic.

Patient 2 presented with fever on February 5 th and the maximum temperature was $38.4^{\circ} \mathrm{C}$. No respiratory or gastrointestinal symptoms occurred during the onset. Fever lasted for 3 days and after that she remained asymptomatic.

\subsection{Laboratory and imaging examination}

Chest CT images of patient 1 on February 5th, 2020 showed multiple ground-glass opacity (fig. 2). Lymphocyte proportion decreased on the day of diagnosis and significantly increased after patient 1 was hospitalized. C-reactive protein level had a slight elevation on the day of diagnosis and turned normal soon. Serum SARS-CoV-2 antibody increased significantly on hospitalized day while decreasing quickly (table 1). SARS-CoV-2 nucleic acid tests of nasopharyngeal swab of patient 1 were negative. Nucleic acid of rectal swab was positive on February 5th and didn't turn negative until March 21st. The $\mathrm{Ct}$ value and viral load of rectal specimen during hospitalization was detailed in fig 3 . The mean viral load of feces was $3.53 \log 10$ copies $/ \mathrm{mL}$.

On February 9th, chest CT images of patient 2 showed small spot-like and fuzzy shadow (fig. 2). White blood cell count was reduced on the day of diagnosis while lymphocyte proportion was increased throughout the course of illness. C-reactive protein was normal and serum SARS-CoV-2 antibody was

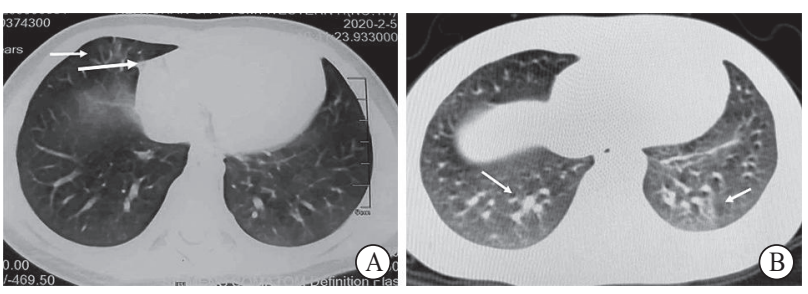

Fig. 2 Chest computed tomographic images of patients A: multiple ground-glass opacity and stripe shadow (arrow) (patient 1); B: small spot-like and fuzzy shadow (arrow) (patient 2)

increased (table 1). The SARS-CoV-2 nucleic acid test of nasopharyngeal swab of patient 2 was positive on February 9th and turned negative on February 19th, while the nucleic acid tests of rectal swabs persisted positive from February 16th to March 25th. The Ct value and viral load of nasopharyngeal and rectal specimen during hospitalization were detailed in figure 3. The mean viral load of feces was $5.32 \log 10$ copies/ $\mathrm{mL}$.

\subsection{Treatment}

Patient 1 was treated with piperacillin sodium and tazobactam sodium for five days at the beginning of illness, and received oral oseltamivir for five days and azithromycin for 4 courses because of the positive results of mycoplasma pneumonia and influenza A virus. During hospitalization, she was given oral ribavirin and subsequently treated with traditional Chinese medicine until nucleic acid turned negative. 
Table 1 Summary of laboratory examination results of patient 1 and patient 2

\begin{tabular}{|c|c|c|c|c|c|c|c|c|c|}
\hline & \multirow{2}{*}{$\begin{array}{l}\text { Reference } \\
\text { range }\end{array}$} & \multicolumn{2}{|c|}{ Diagnosed day } & \multicolumn{2}{|c|}{ Hospitalized day } & \multicolumn{2}{|c|}{$\begin{array}{c}\text { During } \\
\text { hospitalization }\end{array}$} & \multicolumn{2}{|c|}{ Recovery day } \\
\hline & & Patient 1 & Patient 2 & Patient 1 & Patient 2 & Patient 1 & Patient 2 & Patient 1 & 1 Patient 2 \\
\hline White blood cell count, $\times 10^{9} / \mathrm{L}$ & $4.00-10.00$ & 9.29 & $3.93 \downarrow$ & 7.84 & NA & 7.93 & 5.61 & 9.30 & 5.14 \\
\hline Neutrophil ratio, $\%$ & $50.00-70.00$ & $76.80 \uparrow$ & $10.40 \downarrow$ & 17.7 & & $28.9 \downarrow$ & $21.50 \downarrow$ & 67.60 & $22.3 \downarrow$ \\
\hline Lymphocyte ratio, $\%$ & $20.00-40.00$ & $17.50 \downarrow$ & $82.20 \uparrow$ & $73.10 \uparrow$ & NA & $61.5 \uparrow$ & $72.90 \uparrow$ & 20.10 & $72.4 \uparrow$ \\
\hline $\mathrm{CD} 3+$ lymphocyte ratio, $\%$ & $57.90-72.70$ & NA & NA & $50.06 \downarrow$ & NA & NA & NA & $50.23 \downarrow$ & $81.86 \uparrow$ \\
\hline CD4+ lym & $31.70-45.70$ & NA & NA & $27.69 \downarrow$ & NA & NA & NA & $23.04 \downarrow$ & $49.11 \uparrow$ \\
\hline C-reactive & $0.00-10.00$ & $11.50 \uparrow$ & 0.30 & 0.10 & NA & 0.20 & 0.20 & 3.30 & 0.20 \\
\hline Procalcitonin, $\mathrm{ng} / \mathrm{mL}$ & $0.02-0.05$ & NA & NA & $<0.02$ & NA & NA & NA & 0.04 & NA \\
\hline Erythrocyte sedimentation rate, $\mathrm{mm} / \mathrm{H}$ & $0.00-20.00$ & NA & NA & 2.00 & NA & NA & NA & 3.00 & NA \\
\hline Glutamic-pyruvic transaminase, U/L & $\leq 33.00$ & 9.00 & 13.00 & 12.00 & NA & 18.00 & 12.00 & 15.00 & 12.00 \\
\hline Interleukin-6, pg/mL & $<7.00$ & NA & NA & $<1.5$ & NA & NA & $<1.50$ & NA & $<1.50$ \\
\hline Serum SARS-CoV-2 antibody IgM, AU/mL & $\leq 10.00$ & NA & NA & $669.85 \uparrow$ & NA & $59.02 \uparrow$ & $11.96 \uparrow$ & $19.81 \uparrow$ & $10.32 \uparrow$ \\
\hline Serum SARS-CoV-2 antibody IgG, AU/mL & $\leq 10.00$ & NA & NA & $244.22 \uparrow$ & NA & $243.23 \uparrow$ & $173.49 \uparrow$ & $121.43 \uparrow$ & $120.06 \uparrow$ \\
\hline
\end{tabular}

NA: not available

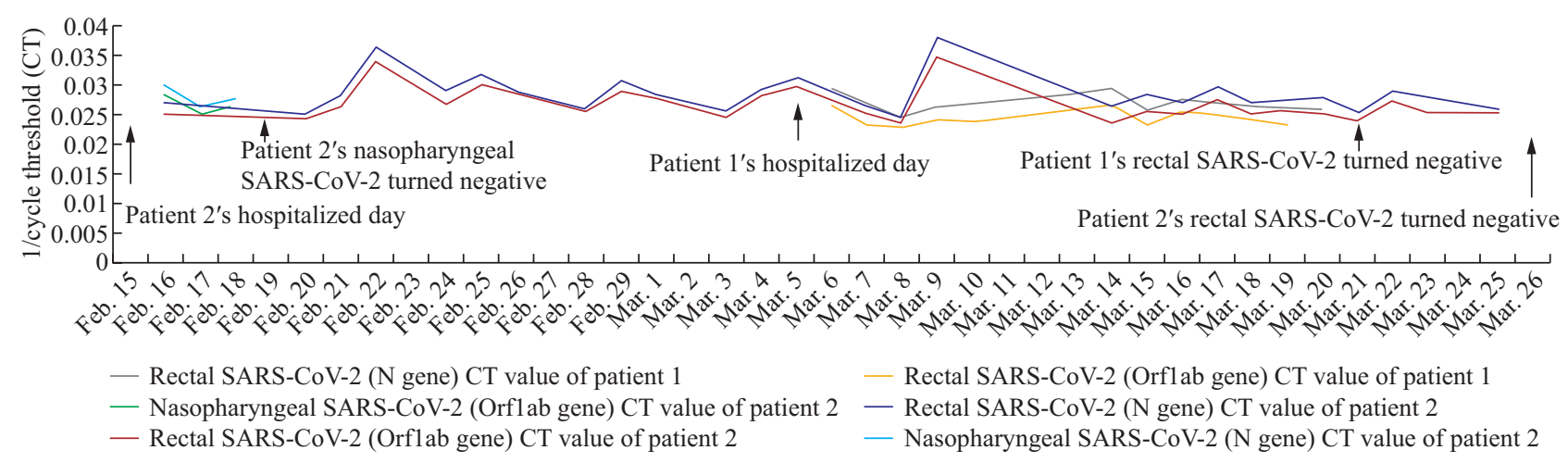

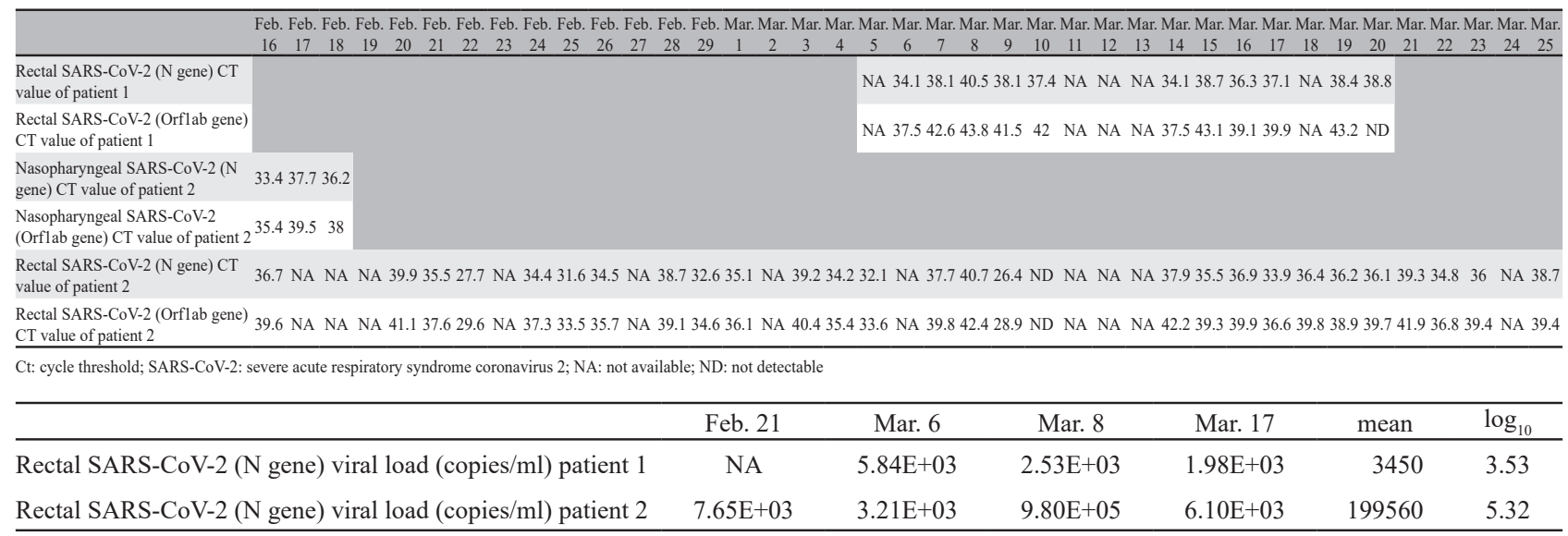

Fig. 3 The Ct value of nucleic acid of rectal and nasopharyngeal swab and feces viral load of in patient 1 and patient 2

Patient 2 was treated alternatively with oral abidor and ribavirin (each for 7 days) after diagnosis. From March 21st she was given Lianhua Qingwen Capsule and traditional Chinese medicine until nucleic acid turned negative.

\section{DISCUSSION}

In this study, we collected two pediatric cases of moderate COVID-19, analyzed their epidemiological data and monitored the $\mathrm{Ct}$ value of rectal specimen and the viral loads. The mean viral load of rectal swabs of the two patients was $3.53 \log _{10}$ and $5.32 \log _{10}$ copies/ $\mathrm{mL}$, and it took 45 and 39 days for viral shedding, respectively. Previous study reported that children with COVID-19 carried a long-term feces viral shedding ${ }^{[12]}$, which is in agreement with our findings. Attentions were put on whether the feces viral shedding could become the source of aerosol and contamination in environmental surfaces to spread virus, which may contribute to the clusters of infection ${ }^{[12]}$. It is reported that virus isolation from stool samples was 
never successful if the viral load $<10^{6}$ copies $/ \mathrm{mL}^{[13]}$. In our study, the $\mathrm{Ct}$ value of the two patients seemed to indicate the unlikelihood of transmission from pediatric patients, especially during the viral shedding of rectal specimen. More studies are needed to definite the virulence of feces virus.

The epidemiological information further confirmed our inference. In these two cases, the two patients were in close contact with their family members from the incubation to progression of COVID-19, without any effective protective measures. But the close contacts, including the elderly, the middle-aged and children, were not infected. In combination with the viral load and epidemiological manifestation, we have reason to infer that toddlers with moderate COVID-19 did not spread the SARS-CoV-2 to adults and other children. Another epidemiologic analysis of a critical ill infant with COVID-19 also suggests the unlikelihood of transmission of COVID-19 even from critical infants to adults ${ }^{[14]}$. In summary, the reopening of kindergartens will not increase the risk of SARS-CoV-2 spread. More studies are needed to discuss whether transmission of COVID-19 in adolescents is different from that in toddlers and infants.

In a word, although there are still new sporadic local cases and imported cases across the country and this situation may last for a long time, the opening of kindergartens will not increase the risk of epidemic transmission. However, this does not mean to relax vigilance. Instead, the focus of epidemic prevention and control in kindergartens should be placed on the monitoring of adults whom children are in close contact with, including parents and kindergarten staff, etc. On the one hand, the body temperature and physical condition of kindergarten staff and the parents who pick up the students every day should be monitored daily. It would be even better if they can provide negative reports of SARS-CoV-2 nucleic acid tests. On the other hand, kindergartens should implement closed management, prevent social personnel from entering the kindergartens and register the parents who pick up the students. In addition, it's also important to conduct epidemic prevention and control education for students and parents, including developing good living habits on personal hygiene, avoiding taking public transportation on the way to and from kindergarten and so on. Only with the joint efforts of kindergartens and parents can children learn and grow up safely and healthily in the period of regular epidemic prevention and control.

\section{Conflict of Interest Statement}

The authors declare that there is no conflict of interest with any financial organization or corporation or individual that can inappropriately influence this work.

\section{REFERENCES}

1 Cucinotta D, Vanelli M. WHO Declares COVID-19 a Pandemic. Acta biomed, 2020,91(1):157-160

2 Velavan TP, Meyer CG. The COVID-19 epidemic. Trop Med Internat Health, 2020,25(3):278-280

3 Paules CI, Marston HD, Fauci AS. Coronavirus Infections-More Than Just the Common Cold. JAMA, 2020:323(8):707-708

4 Bai Y, Yao L, Wei T, et al. Presumed Asymptomatic Carrier Transmission of COVID-19. JAMA, 2020, 323(14):1406-1407

5 Chan JFW, Yuan S, Kok KH, et al. A familial cluster of pneumonia associated with the 2019 novel coronavirus indicating person-to-person transmission: a study of a family cluster. Lancet, 2020,395(10223):514-523

6 Ludvigsson JF. Systematic review of COVID-19 in children shows milder cases and a better prognosis than adults. Acta Paediatr, 2020,109(6):1088-1095

7 Wei M, Yuan J, Liu Y, et al. Novel Coronavirus Infection in Hospitalized Infants Under 1 Year of Age in China. JAMA, 2020,323(13):1313-1314

8 Lu X, Zhang L, Du H, et al. SARS-CoV-2 Infection in Children. New Engl J Med, 2020,382(17):1663-1665

9 Tezer H, Bedir Demirdag T. Novel coronavirus disease (COVID-19) in children. Turk Med Scik, 2020,50(SI1):592-603

10 Zimmermann P, Curtis N. COVID-19 in Children, Pregnancy and Neonates: A Review of Epidemiologic and Clinical Features. Pediatr Infect Dis J, 2020, 39(6):469-477

$11 \mathrm{Bi}$, Wu Y, Mei S, et al. Epidemiology and transmission of COVID-19 in 391 cases and 1286 of their close contacts in Shenzhen, China: a retrospective cohort study. Lancet Infect Dis, 2020,20(8):911-919

$12 \mathrm{Xu} \mathrm{Y,} \mathrm{Li} \mathrm{X,} \mathrm{Zhu} \mathrm{B,} \mathrm{et} \mathrm{al.} \mathrm{Characteristics} \mathrm{of} \mathrm{pediatric}$ SARS-CoV-2 infection and potential evidence for persistent fecal viral shedding. Nat Med, 2020,26(4):502505

13 Wölfel R, Corman VM, Guggemos W, et al. Virological assessment of hospitalized patients with COVID-2019. Nature, 2020,581(7809):465-469

14 Qiu L, Jiao R, Zhang A, et al. A Case of Critically Ill Infant of Coronavirus Disease 2019 with Persistent Reduction of $\mathrm{T}$ Lymphocytes. Pediatr Infect Dis J, 2020,39(7):e87-e90

(Received Aug. 16, 2020; accepted Sep. 12, 2020) 